REGULAR ARTICLE

\title{
SCREEN HOUSE ASSESSMENT OF NEEM-FORTIFIED CASSAVA PEEL POWDER FOR CONTROLLING NEMATODES AND YIELD IMPROVEMENT OF SUGARCANE (SACCHARUM OFFICINARUM)
}

\author{
S. SLOMON ${ }^{*}$, S. O. AFOLAMI², T. O. S. POPOOLA3 3 , J. J. ATUNGWU3, S. ODEYEMI3, \\ F. Y. DARAMOLA4
}

${ }^{1}$ Nigeria Agricultural Quarantine Service, P. M. B 285 Abuja, Nigeria

${ }^{2}$ Augustine University, P. M. B. 1010, Lagos, Nigeria

3Department of Crop Protection, Federal University of Agriculture, Abeokuta, Nigeria

4Department of Biological Sciences, Covenant University, P. M. B 1023, Ota, Nigeria

\begin{abstract}
Screen house trials for the assessment of Neem-fortified cassava peels for control of plant-parasitic nematodes and yield improvement of sugarcane (Saccharum officinarum) were conducted in Moor plantation, Ibadan, Southwest Nigeria. Cassava peel powder (CPP) solely and in combination with Neem Leaf Powder (NLP) or Neem Seed Powder (NSD) at 10ogCPP/10 Litre soil, $90 \mathrm{CPP}+30 \mathrm{gNSP} / 10$ Litre soil, $80 \mathrm{gCPP}+20 \mathrm{NSP} / 10$ Litre soil, $70 \mathrm{CPP}+30 \mathrm{NNS} / 10$ Litre of soil, $90 \mathrm{CPP}+10 \mathrm{gNLP} / 10$ Litre soil, $80 g \mathrm{CPP}+20 \mathrm{gNLP} / 10$ Litre soil, $70 \mathrm{OCPP}+30 g N L P / 10$ Litre soil were incorporated into potted naturally infested soil in the screen house, at $14 \mathrm{~d}$ before planting. All treatments significantly $(\mathrm{p}<0.05)$ reduced plant-parasitic nematode population. Soil treatment with Cassava Peel Powder in combination with Neem Leaf Powder at 70gCPP +30 ogLP/10 Litre of soil recorded the most effective control for soil and root nematodes associated with sugarcane in the two screen house trials. The soil amendment also supported the good vegetative growth and yield of sugarcane, which is an indication of its capability to improve soil fertility.
\end{abstract}

Keywords: Sugar cane, Cassava Peel Powder, Neem Leaf Powder, Nematode population, Soil amendment

\section{INTRODUCTION}

Sugarcane (Saccharum officinarum,) is a large tropical grass that is grown commercially for sugar production [14]. Nematode infection in sugarcane is common and endo-parasitic and ecto-parasitic nematodes described world-wide from plant's roots and rhizosphere [29]. Plantparasitic nematodes are main threat to crops [17]. Meloidogyne and Pratylenchus species are among the most frequently reported endo-parasitic phytonematodes that are highly pathogenic on sugarcane [7]. The root knot nematodes ( $M$. incognita and $M$. javanica) alongside with Pratylenchus zea have caused productivity losses [9].

Sugarcane is grown by thousands of local farmers in Nigeria [1]. It is cultivated for its chewable pulps, which provide a source of income to peasant farmers and also commercially for sugar production [18]. The soft chewing cane (Bekki variety) is usually grown as a continuous monoculture in many Nigerian farms, with not more than a few months interval between the removal of old ratoon crop and replanting of the field. This condition tends to favour the development of relatively large populations of nematode species [17] and consequently reduction in crop yield.

Nematicides have been used in crop production for inhibiting infection [2]. Despite the significant progress recorded in the use of synthetic pesticide for nematode control, their uses remain a major concern to human health and environment. Synthetic pesticides alone account for more than 20,000 deaths annually, which occur mostly in developing countries [22]. The associated prohibitive cost, scarcity and even lack of the required basic knowledge for the right application of these chemicals are some of the limitations to the use of nematicides in many developing countries where a large percentage of resource poor farmers do not have access to formal education.

Plant extracts of Neem (Azadirachta indica) have

Received 14 February 2018; Accepted 22 May 2018

*Corresponding Author

\section{S. Slomon}

Nigeria Agricultural Quarantine Service, P. M. B 285 Abuja, Nigeria

Email: solomonpqs@yahoo.com

(T) This article is open access and licensed under the terms of the Creative Commons Attribution License (http://creativecommons.org/licenses/by/4.0/) which permits unrestricted, use, distribution and reproduction in any medium, or format for any purpose, even commercially provided the work is properly cited. Attribution - You must give appropriate credit, provide a link to the license, and indicate if changes were made. 
produced viable and environmentally benign alternatives to the use of nematicides. They are cheaper, easily degradable in soil with no harmful residues and toxic effects on host plants and humans [5]. The extracts of neem from fruits, seeds, twigs, stem bark, leaves have yielded insect anti-feedant, nematicidal, fungicidal, and bactericidal properties, which have gained wide applications in commercial agriculture [27]. In Nigeria, $A$. indica leaf extract and cassava (M. esculenta Crantz) peels have been reported $[11,12]$ to be effective in controlling Pratylenchus brachyurus on Zea mays.

Limited literature is available on the effect of neem-fortified cassava peel powder on the management of plant-parasitic nematodes in Nigeria. Therefore, the objective of this study is to evaluate the efficacy of neem-fortified cassava peel powder for the control of plant-parasitic nematodes associated with sugarcane in the screen house and its subsequent effect on the growth and yield of sugarcane.

\section{MATERIALS AND METHODS}

\section{Source of planting material}

Stem cuttings Saccharum officinarum cv. Bekki were obtained from some local farmers in Moor plantation, Ibadan, South-west, Nigeria. Ripe neem fruits and fresh cassava root peels, which were used as soil amendments for the screenhouse trials, were also obtained from Moor plantation in Ibadan, South-west, Nigeria.

\section{Experimental site}

Two screen house trials to determine the suppressive effects of neem-fortified cassava peel on nematode population and yield of sugarcane were carried out in 2013 and 2014, at the Nigeria Agricultural Quarantine Service (NAQS) Moor Plantation, Ibadan, South-west, Nigeria (Latitude $7.22{ }^{\circ} \mathrm{N}$, Longitude $3.58^{\circ} \mathrm{E}$ and $122 \mathrm{~m}$ above sea level). The annual rainfall of Ibadan ranged between $1200-2500 \mathrm{~mm}$, and was distributed over a period of about 7 mo (April to October). The average daily temperature range was $26-30^{\circ} \mathrm{C}$.

\section{Collection of soil sample for initial nematode analysis}

Soil samples for nematode analysis were collected from each experimental plot prior to planting, and at harvest. Top soil that is naturally infested with plant-parasitic nematodes was collected from the Fadama area of National Cereal Research Institute (NCRI) research field at Moor Plantation, Ibadan with the aid of a hand trowel to a depth of about $30 \mathrm{~cm}$ and radius of $25 \mathrm{~cm}$ from the base of plants. The soil samples were bulked and thoroughly mixed using a shovel. Five $250 \mathrm{ml}$ sub-samples of soil were taken from the bulk and thoroughly mixed again before weighing out two $200 \mathrm{~g}$ samples for nematode extraction.

\section{Nematode extraction to determine initial nematode type and population}

The Extraction Tray method of [33] was used for the nematode extraction to determine the presence, initial population and types of the nematodes in the soil. Soil nematodes were extracted from a $200 \mathrm{ml}$ sub-sample of soil, using the [33] tray modification of Baermann extraction method. The nematode species were identified with the aid of a compound microscope using the pictorial key of [16] as a guide. The number of each nematode species per $200 \mathrm{ml}$ of soil sample was counted with the aid of a tally counter under a stereomicroscope.

\section{Soil analysis for physico-chemical properties}

Soil samples for the screen house experiments were subjected to chemical and physical analysis in the laboratory to determine the physico-chemical properties of the soil before treatment application. Organic carbon and organic matter content were determined using the dichromate oxidation method, total Nitrogen was determined using a micro-Kjedahl apparatus, available P, $\mathrm{Ca}^{2+}$ and $\mathrm{Mg}^{2+}$ were determined using an atomic absorption spectrophotometer while exchangeable $\mathrm{Na}^{+}$and $\mathrm{K}^{+}$were determined with a flame photometer.

\section{Preparation of neem formulations and cassava- peel powder}

Ripe neem fruits were processed to remove the mucilaginous pericarp. The seeds obtained were air-dried for about three weeks to a moisture content of about $13 \%$ before grinding to granules and then sieved with a $2 \mathrm{~mm}$ diameter sieve. Green leaves of neem were also collected and air-dried for $3 \mathrm{w}$ before grinding to powder using heavy duty grinder.

Fresh cassava root peels were carefully washed in tap water, air-dried for approximately $3 \mathrm{w}$ until the moisture content was less than $13 \%$, crushed and converted into powder using a grinder as earlier described. The three plant extracts were placed in polythene bags, labelled appropriately and kept at room temperature in the laboratory.

\section{Experimental design in the screen house}

Soil samples from naturally infested soil were distributed into forty-eight (48) 10-litre buckets. $10 \mathrm{~kg}$ of the infested soil was placed in each 10 litre bucket. Stem cuttings Saccharum officinarum cv. Bekki with two buds each were surface-sterilized in $1.5 \%$ sodium hypochlorite $(\mathrm{NaOCl})$ solution for1o min and later planted in the 48 pots at 1 cutting per pot. The screen house experiments comprise of eight rates of Cassava Peel Powder (CPP), which were applied solely or in combination with Neem Seed Powder (NSP) or Neem Leave Powder (NLP). The treatment applied include; $100 \mathrm{CPP} / 10$ Litre soil, 90 CPP $+30 g N S P / 10$ Litre soil, $80 g \mathrm{CPP}+20$ NSP/10 Litre soil, $70 \mathrm{CPP}+30 \mathrm{gNSP} / 10$ Litre of soil, 90gCPP+10gNLP/10 Litre soil, 8ogCPP+20gNLP/10 Litre soil, $70 \mathrm{CPP}+30 \mathrm{gNLP} / 10$ Litre soil, and control (no amendment). Each treatment was replicated six times and the experiment laid out in a Randomised Complete Block Design (RCBD). The treatments were applied $14 \mathrm{~d}$ prior to planting to allow for proper mineralisation. Each pot was irrigated with $200 \mathrm{ml}$ of water daily for nine months after which the experiment was terminated.

\section{Data collection in the screen house}

At the termination of the experiment, the fresh root weight and shoot weight were recorded per plant. Nematodes were also extracted from root and soil samples for identification and counting. Root samples were observed for galls, cysts and lesions. Root samples were cut transversely with scissors into about 1-2 cm, and $10 \mathrm{~g}$ of root samples from each pot was assayed for nematodes using the [33] tray modification of Baermann technique. Likewise, soil samples from the potted sugarcane plants were sampled for vermiform nematodes using the [33] tray modification of Baermann technique as previously described. 


\section{Data analysis}

The data from the screen house investigation were subjected to analysis of variance using SAS program version 8.1 (SAS 2000) and means were separated using Duncan's New Multiple Range Test (DNMRT).

\section{RESULTS}

\section{Physico-chemical properties of soil}

The physico-chemical characteristic of the naturally infested soils used for the two screenhouse trials is described in table 1 . The soil samples were sandy-loam.

\section{Initial nematode population of plant parasitic} nematodes in the naturally infested soil

Thirteen species of nematodes were recovered from the initial nematode analysis of the naturally infested soil samples. They include; Pratylenchus zeae, Helicotylenchus dihystera, Rotylenchulus reniformis, Meloidogyne spp., Cricinemoides limitaneum, Hoplolaimus indicus, Trichodorous spp., Scutellonema brachyuruma, Tylenchus spp., Hemicyclophora spp., Longidorus spp., Monochids and Rhabditis (see table 2).

Suppressive effects of neem-fortified cassava peel powder on plant parasitic nematodes of sugarcane

Soil treatment with Cassava Peel Powder (CPP) in combination with Neem Leaf Powder (NLP) at $70 g C P P+30 g N L P / 10$ Litre of soil gave the most effective control for soil and root nematodes associated with sugarcane in the two screen house trials (tables 3-6). Significantly lower population densities of plant-parasitic nematodes $(\mathrm{p}<0.05)$ were observed in soils treated with CPP alone and in combination with either NSP or NLP as compared with the control (table 3 and 4). Lower population of the endo-parasitic nematodes were also recovered from the roots of sugarcane plants amended with Neem-fortified Cassava Peel powder at $70 g C P P+30 g N L P / 10$ Litre of soil (5 and 6). At harvest, the soil density of plant-parasitic nematodes (PPN) recovered under sugarcane from the unamended pots was significantly $(\mathrm{p}<0.05)$ higher than the density of nonparasitic nematodes (NPN) recorded for the two screen house trials (fig. 1 and 2). The reverse was however the case in the neem-fortified cassava peel pots where lower number of PPN was recorded on the sugarcane plants.

\section{Effects of neem-fortified cassava peel powder on} sugarcane yield

There were significant treatment effects on the shoot weight, root weight, height and girth of the sugarcane plants in the two screen house trials (Tables 7 and 8). Soil amendment with cassava peel powder and neem leaf powder combined at $70 \mathrm{CPP}+30 \mathrm{gNLP} / 10$ Litre resulted in significant $(\mathrm{p}<0.05)$ increase in the shoot weight, root weight, stalk height and stalk girth of the sugarcane plants (Tables 7 and 8). Significantly low shoot and root weights, stalk height and stalk girth were recorded on the unamended pots. There was however no significant treatment effect on the number of tillers of the sugarcane plants.

Table 1: Physical and chemical characteristic of the soil of the experimental pots before planting

\begin{tabular}{lll}
\hline Soil property & $\mathbf{2 0 1 3}$ & $\mathbf{2 0 1 4}$ \\
\hline $\mathrm{Na}(\mathrm{cmol} / \mathrm{kg})$ & 0.99 & 1.07 \\
$\mathrm{~K}(\mathrm{cmol} / \mathrm{kg})$ & 0.46 & 0.53 \\
$\mathrm{Ca}(\mathrm{cmol} / \mathrm{kg})$ & 1.93 & 2.41 \\
$\mathrm{Mg}(\mathrm{cmol} / \mathrm{kg})$ & 1.73 & 1.96 \\
$\% \mathrm{O} . \mathrm{C}$ & 3.15 & 3.49 \\
$\% \mathrm{~N}$ & 0.35 & 0.45 \\
$\mathrm{Ppm}(\mathrm{Av} . \mathrm{p})$ & 6.85 & 8.36 \\
$\mathrm{Zn}(\mathrm{ppm})$ & 3.86 & 4.01 \\
$\mathrm{Co}(\mathrm{ppm})$ & 1.36 & 1.26 \\
$\mathrm{Mn}(\mathrm{ppm})$ & 3.84 & 4.99 \\
$\mathrm{pH}$ & 6.15 & 6.25 \\
\hline
\end{tabular}

Table 2: Initial soil densities of pant-parasitic nematodes in a naturally infested field cleared for sugarcane cutting establishment in 2013 and 2014

\begin{tabular}{lll}
\hline Nematode species & population 2oo g/soil & population 2oo g/soil \\
\hline Pratylenchus zeae & $22.67 \mathrm{bc}$ & $25.33 \mathrm{~b}$ \\
Helicotylenchus dihystera & $27.00 \mathrm{bc}$ & $23.00 \mathrm{bc}$ \\
Rotylenchulus reniformis & $21.33 \mathrm{~cd}$ & $18.33 \mathrm{~cd}$ \\
Meloidogyne sp. & $27.33 \mathrm{~b}$ & $22.00 \mathrm{bc}$ \\
Cricinemoides limitaneum & $2.67 \mathrm{~g}$ & $4.33 \mathrm{e}$ \\
Hoplolaimus indicus & $1.33 \mathrm{~g}$ & $1.33 \mathrm{e}$ \\
Trichodorous spp & $3.67 \mathrm{~g}$ & $6.33 \mathrm{e}$ \\
Scutellonema brachyuruma & $0.00 \mathrm{~g}$ & $1.00 \mathrm{e}$ \\
Tylenchus spp. & $13.67 \mathrm{ef}$ & $17.67 \mathrm{~cd}$ \\
Hemicyclophora spp. & $10.67 \mathrm{f}$ & $7.00 \mathrm{e}$ \\
Longidorus spp. & $16.67 \mathrm{de}$ & $13.00 \mathrm{~d}$ \\
Monochid species & $80.00 \mathrm{a}$ & $79.00 \mathrm{a}$ \\
Rhabditis spp & $13.67 \mathrm{ef}$ & $13.67 \mathrm{~d}$ \\
\hline
\end{tabular}


Table 3: Mean nematode population densities per $200 \mathrm{~g}$ soil of Saccharum officinarum cv. Bekki at harvest under different combinations of Neem-fortified Cassava Peel powder treatments in the screen house in 2013

\begin{tabular}{|c|c|c|c|c|c|c|c|c|c|c|c|c|c|}
\hline Treatment & $\begin{array}{l}\text { z. } \\
\text { zea }\end{array}$ & $\begin{array}{l}\text { H. } \\
\text { dihystera }\end{array}$ & $\begin{array}{l}\text { M.. } \\
\text { incognita }\end{array}$ & $\begin{array}{l}\text { Trichodorus } \\
\text { spp }\end{array}$ & $\begin{array}{l}\text { Longidorus } \\
\text { Spp }\end{array}$ & $\begin{array}{l}R . \\
\text { reniformis }\end{array}$ & $\begin{array}{l}\text { Tylenchus } \\
\text { spp }\end{array}$ & $\begin{array}{l}\text { H. } \\
\text { indicus }\end{array}$ & $\begin{array}{l}\text { C. } \\
\text { limitaneum }\end{array}$ & $\begin{array}{l}\text { Hemicyclophora } \\
\text { spp }\end{array}$ & $\begin{array}{l}\text { S. } \\
\text { brachyurus }\end{array}$ & Mononchids & Rhabiditis \\
\hline Control & $23.67 \mathrm{a}$ & $15.50 \mathrm{a}$ & $16.83 a$ & $1.83 \mathrm{ab}$ & $4.00 a$ & $7.17 \mathrm{a}$ & $3,50 a$ & $1.00 \mathrm{a}$ & $1.33^{a}$ & $1.17 \mathrm{ab}$ & $0.33 a$ & $61.33 a$ & $3.67 \mathrm{a}$ \\
\hline $\begin{array}{l}\text { CPP } 100 \\
\text { CPP + NS }\end{array}$ & $14.17 \mathrm{~b}$ & 8.oob & $13.33 \mathrm{ab}$ & $2.50 \mathrm{a}$ & $3 \cdot 33 \mathrm{ab}$ & $3.5 \mathrm{ob}$ & $2.00 a b$ & $0.00 \mathrm{a}$ & $0.83 a b$ & $0.50 a b$ & $0.17 \mathrm{ab}$ & $53.83 \mathrm{~b}$ & $5.00 \mathrm{a}$ \\
\hline $\begin{array}{l}(9: 1) \\
\mathrm{CPP}+\mathrm{NS}\end{array}$ & $12.67 \mathrm{bcd}$ & $7.67 \mathrm{bc}$ & $13.83 \mathrm{ab}$ & $1.67 \mathrm{abc}$ & $2.17 \mathrm{abc}$ & $4.00 \mathrm{~b}$ & $2.67 \mathrm{a}$ & $0.17 \mathrm{a}$ & $0.50 a b$ & $1.67 \mathrm{a}$ & $0.00 \mathrm{~b}$ & $49.83 \mathrm{~b}$ & $5.00 \mathrm{a}$ \\
\hline $\begin{array}{l}(8: 2) \\
\mathrm{CPP}+\mathrm{NL}\end{array}$ & $12.17 \mathrm{bcd}$ & $7.83 \mathrm{bc}$ & $12.83 \mathrm{abc}$ & $1.67 \mathrm{abc}$ & $1.17 \mathrm{abc}$ & $3.33 \mathrm{~b}$ & $2.17 \mathrm{ab}$ & $0.17 \mathrm{a}$ & $0.50 a b$ & $0.83 \mathrm{ab}$ & o.oob & $46.00 b c$ & $3.50 \mathrm{Oa}$ \\
\hline $\begin{array}{l}(9: 1) \\
\mathrm{CPP}+\mathrm{NS}\end{array}$ & $13.5 \mathrm{obc}$ & $8.17 \mathrm{~b}$ & $13.17 \mathrm{ab}$ & $2.33 a$ & $2.17 \mathrm{abc}$ & $3.00 \mathrm{~b}$ & $3.17 \mathrm{a}$ & $0.00 \mathrm{a}$ & $0.33 \mathrm{~b}$ & $0.50 a b$ & o.oob & $51.33 \mathrm{~b}$ & $2.50 a b$ \\
\hline $\begin{array}{l}(8: 2) \\
\mathrm{CPP}+\mathrm{NL}\end{array}$ & $11.83 \mathrm{bcd}$ & $5.17 \mathrm{bc}$ & $11.33 \mathrm{bc}$ & $1.67 \mathrm{abc}$ & $1.17 \mathrm{abc}$ & $2.83 \mathrm{~b}$ & $2.5 \mathrm{Oab}$ & $0.00 a$ & $0.50 a b$ & $0.33 \mathrm{~b}$ & $0.00 b$ & $39.33 \mathrm{c}$ & $4.50 \mathrm{O}$ \\
\hline$(7: 3)$ & $10.00 \mathrm{~cd}$ & $5.00 \mathrm{bc}$ & $8.50 \mathrm{c}$ & $0.33 \mathrm{bc}$ & $1.17 \mathrm{abc}$ & $2.17 \mathrm{bc}$ & $1.5 \mathrm{Oab}$ & $0.00 \mathrm{a}$ & $0.50 a b$ & o.oob & o.oob & $46.67 \mathrm{bc}$ & $2.83 \mathrm{ab}$ \\
\hline Carbofuran & $5.50 \mathrm{e}$ & $2.5 \mathrm{~cd}$ & $3.17 \mathrm{~d}$ & $0.33 \mathrm{bc}$ & $0.5 \mathrm{obc}$ & $1.17 \mathrm{bc}$ & $1.17 \mathrm{ab}$ & o.ooa & $0.50 a b$ & $0.17 \mathrm{~b}$ & o.oob & $22.17 \mathrm{~d}$ & $1.83 \mathrm{ab}$ \\
\hline Sterilized soil & $0.00 f$ & o.ood & o.ood & $0.00 c$ & $0.00 \mathrm{c}$ & $0.00 c$ & $0.00 \mathrm{~b}$ & $0.00 \mathrm{a}$ & o.oob & $0.00 \mathrm{~b}$ & $0.00 \mathrm{~b}$ & o.ood & $0.00 \mathrm{~b}$ \\
\hline
\end{tabular}

*Means followed by the same letter in the same column do not differ significantly according to Duncan Multiple Range Test ( 5 \%).

Table 4: Mean nematode population densities per $200 \mathrm{~g}$ soil of Saccharum officinarum cv. Bekki at harvest under different combinations of Neem-fortified Cassava Peel powder treatments in the screen house in 2014

\begin{tabular}{|c|c|c|c|c|c|c|c|c|c|c|c|c|c|}
\hline Treatment & $\begin{array}{l}P . \\
\text { zea }\end{array}$ & $\begin{array}{l}\text { H. } \\
\text { Dihystera }\end{array}$ & $\begin{array}{l}\text { M.. } \\
\text { incognita }\end{array}$ & T.spp & $\begin{array}{l}\text { Longidorus } \\
\text { Spp }\end{array}$ & $\begin{array}{l}R . \\
\text { reniformis }\end{array}$ & $\begin{array}{l}\text { Tylenchus } \\
\text { spp }\end{array}$ & $\begin{array}{l}\mathrm{H} . \\
\text { indicus }\end{array}$ & $\begin{array}{l}\text { C. } \\
\text { limitaneum }\end{array}$ & $\begin{array}{l}\text { Hemicyclophora } \\
\text { spp }\end{array}$ & $\begin{array}{l}\text { S. } \\
\text { brachyurus }\end{array}$ & Mononchids & Rhabiditis \\
\hline Control & $32.50 \mathrm{a}$ & $5.75 \mathrm{a}$ & 28.50a & $0.50 \mathrm{a}$ & $3.00 \mathrm{a}$ & $1.25 \mathrm{ab}$ & $2.25 \mathrm{a}$ & $1.25 \mathrm{a}$ & $1.33^{a}$ & $1.00 \mathrm{a}$ & $0.00 \mathrm{a}$ & $69.25 \mathrm{a}$ & $1.75 a$ \\
\hline CPP 100 & $19.50 \mathrm{~b}$ & $4.50 \mathrm{ab}$ & $22.75^{b}$ & $1.50 \mathrm{a}$ & $2.75^{\mathrm{ab}}$ & $2.50 \mathrm{ab}$ & $2.00 \mathrm{ab}$ & $1.75^{a}$ & $0.83 a b$ & $1.00 \mathrm{a}$ & $0.00 \mathrm{a}$ & $64.25 \mathrm{a}$ & $2.25 \mathrm{a}$ \\
\hline $\mathrm{CPP}+\mathrm{NS}(9.1)$ & $16.00 \mathrm{c}$ & $4.00 \mathrm{ab}$ & $17.50 \mathrm{c}$ & $1.00 \mathrm{a}$ & $2.00 \mathrm{abc}$ & $2.25 \mathrm{ab}$ & $1.50 a b$ & $1.00 \mathrm{a}$ & $0.50 a b$ & $0.75 \mathrm{ab}$ & $0.00 \mathrm{a}$ & $49.7 \mathrm{sb}$ & $1.75^{a}$ \\
\hline $\mathrm{CPP}+\mathrm{NS}(8.2)$ & $13.25 \mathrm{c}$ & $2.75 \mathrm{bc}$ & $14.25 \mathrm{~cd}$ & $1.00 \mathrm{a}$ & $1.75 \mathrm{abc}$ & $2.75 \mathrm{a}$ & $1.50 a b$ & $1.75^{a}$ & $0.50 a b$ & $0.75 \mathrm{ab}$ & $0.00 \mathrm{a}$ & $44.5 \mathrm{obc}$ & $1.25 \mathrm{a}$ \\
\hline $\mathrm{CPP}+\mathrm{NL}(9.1)$ & $13.25 \mathrm{c}$ & $2.75 \mathrm{bc}$ & $13.75 \mathrm{~cd}$ & $0.75 a$ & $1.75 \mathrm{abc}$ & $2.00 \mathrm{ab}$ & $0.75^{a b}$ & $1.25 \mathrm{a}$ & $0.33 \mathrm{~b}$ & $0.50 \mathrm{ab}$ & $0.00 \mathrm{a}$ & $40.00 c$ & $0.75 a$ \\
\hline $\mathrm{CPP}+\mathrm{NS}(7.3)$ & $9.75 \mathrm{~d}$ & $1.75 \mathrm{c}$ & 11.75 de & $0.75 \mathrm{a}$ & $1.25 \mathrm{bc}$ & $1.75 \mathrm{ab}$ & $0.75 \mathrm{ab}$ & $1.00 \mathrm{a}$ & $0.33 \mathrm{~b}$ & $0.25 \mathrm{ab}$ & $0.00 \mathrm{a}$ & $32.75 \mathrm{~d}$ & $1.50 \mathrm{a}$ \\
\hline $\mathrm{CPP}+\mathrm{NL}(8.2)$ & $8.75 \mathrm{~d}$ & $1.50 \mathrm{c}$ & $9.00 \mathrm{ef}$ & $0.50 \mathrm{a}$ & $0.75 \mathrm{c}$ & $1.00 \mathrm{~b}$ & $0.25 \mathrm{~b}$ & $0.75 a$ & $0.50 a b$ & $0.00 \mathrm{~b}$ & $0.00 \mathrm{a}$ & $26.75 \mathrm{de}$ & $2.25 \mathrm{a}$ \\
\hline $\mathrm{CPP}+\mathrm{NL}(7.3)$ & $4.75 \mathrm{e}$ & $2.00 \mathrm{c}$ & $9.00 \mathrm{ef}$ & $0.50 \mathrm{a}$ & $0.50 \mathrm{c}$ & $1.50 \mathrm{ab}$ & $0.25 \mathrm{~b}$ & $1.25 \mathrm{a}$ & $0.50 \mathrm{ab}$ & $0.00 \mathrm{~b}$ & $0.00 \mathrm{a}$ & $24.75 \mathrm{e}$ & $1.50 \mathrm{a}$ \\
\hline Sterilized soil & $0.00 \mathrm{f}$ & $0.00 \mathrm{~d}$ & $0.00 \mathrm{~g}$ & $0.00 \mathrm{~b}$ & $0.00 \mathrm{c}$ & $0.00 \mathrm{c}$ & $0.00 \mathrm{~b}$ & $0.00 \mathrm{~b}$ & $0.00 \mathrm{~b}$ & $0.00 \mathrm{~b}$ & $0.00 \mathrm{a}$ & $0.00 \mathrm{f}$ & $0.00 \mathrm{~b}$ \\
\hline
\end{tabular}

*Means followed by the same letter in the same column do not differ significantly according to Duncan Multiple Range Test (5\%).

Table 5: Mean nematode population densities per $10 \mathrm{~g}$ fresh root weight of Saccharum officinarum cv. Bekki at harvest under different combinations of Neem-fortified Cassava Peel powder treatments in the screen house in 2013

\begin{tabular}{|c|c|c|c|c|c|c|c|c|c|}
\hline Treatment & P. zea & $\begin{array}{l}\text { Helicotylenchus } \\
\text { dihystera }\end{array}$ & $\begin{array}{l}\text { Meloidogyne } \\
\text { Incognita }\end{array}$ & $\begin{array}{l}\text { Trichodurus } \\
\text { spp }\end{array}$ & $\begin{array}{l}\text { Longidorus } \\
\text { spp. }\end{array}$ & $\begin{array}{l}\text { Rotylenchulus } \\
\text { reniformis }\end{array}$ & $\begin{array}{l}\text { Tylenchus } \\
\text { spp }\end{array}$ & $\begin{array}{l}\text { Hoplolaimus } \\
\text { indicus }\end{array}$ & $\begin{array}{l}\text { Criconemoides } \\
\text { limitaneum }\end{array}$ \\
\hline Control & $3.67 \mathrm{a}$ & $3.67 a$ & $4.83 a$ & $0.17 \mathrm{a}$ & $0.33^{a}$ & $2.83 a$ & $3.00 \mathrm{a}$ & $0.00 \mathrm{a}$ & $0.17 \mathrm{ab}$ \\
\hline CPP 100 & $3.00 a b$ & $2.17 \mathrm{~b}$ & $3.00 \mathrm{~b}$ & o.ooa & $0.00 b$ & $1.5 \mathrm{ob}$ & $2.00 \mathrm{~b}$ & $0.17 \mathrm{a}$ & o.oob \\
\hline CPP + NS (9:1) & $2.5 \mathrm{obc}$ & $1.67 \mathrm{bc}$ & $2.17 \mathrm{bc}$ & o.ooa & o.oob & $1.67 \mathrm{~b}$ & $1.00 \mathrm{c}$ & $0.00 a$ & o.oob \\
\hline $\mathrm{CPP}+\mathrm{NS}(8: 2)$ & $2.17 \mathrm{bcd}$ & o.83cde & $1.67 \mathrm{c}$ & $0.00 a$ & o.oob & $0.67 \mathrm{~cd}$ & $1.17 \mathrm{c}$ & $0.00 a$ & o.oob \\
\hline $\mathrm{CPP}+\mathrm{NL}(9: 1)$ & $2.17 \mathrm{bcd}$ & $1.33 \mathrm{bc}$ & $2.00 \mathrm{bc}$ & $0.00 a$ & o.oob & $1.33 \mathrm{bc}$ & $1.00 \mathrm{c}$ & $0.00 a$ & $0.33^{a}$ \\
\hline $\mathrm{CPP}+\mathrm{NS}(7: 3)$ & $1.33 \mathrm{~d}$ & o.83cde & $1.17 \mathrm{~cd}$ & $0.00 \mathrm{a}$ & $0.00 \mathrm{~b}$ & $1.33 \mathrm{bc}$ & $1.00 \mathrm{c}$ & $0.00 a$ & $0.00 \mathrm{~b}$ \\
\hline CPP + NL (8:2) & $1.33 \mathrm{~d}$ & $1.17 \mathrm{bcd}$ & $1.67 \mathrm{c}$ & $0.00 a$ & $0.00 b$ & $0.67 \mathrm{~cd}$ & o.5ocd & $0.00 \mathrm{a}$ & $0.00 b$ \\
\hline $\mathrm{CPP}+\mathrm{NL}(7: 3)$ & $1.50 \mathrm{~cd}$ & $0.17 \mathrm{de}$ & $1.00 \mathrm{~cd}$ & $0.00 \mathrm{a}$ & o.oob & $0.67 \mathrm{~cd}$ & $0.33^{\mathrm{cd}}$ & $0.00 \mathrm{a}$ & $0.00 b$ \\
\hline Sterilized soil & o.ooe & o.ooe & o.ood & o.ooa & o.oob & o.ood & o.ood & o.ooa & o.oob \\
\hline
\end{tabular}

*Means followed by the same letter in the same column do not differ significantly according to Duncan Multiple Range Test (5 \%). 
Table 6: Mean nematode population densities per $10 \mathrm{~g}$ fresh root weight of Saccharum officinarum cv. Bekki at harvest under different combinations of Neem-fortified Cassava Peel powder treatments in the screen house in 2014

\begin{tabular}{|c|c|c|c|c|c|c|c|c|c|}
\hline Treatment & P. zea & $\begin{array}{l}H . \\
\text { Dihystera }\end{array}$ & $\begin{array}{l}\text { M. } \\
\text { incognita }\end{array}$ & $\begin{array}{l}\text { Trichodurus } \\
\text { spp. }\end{array}$ & $\begin{array}{l}\text { Longidorus } \\
\text { spp. }\end{array}$ & R.reniformis & $\begin{array}{l}\text { Tylenchus } \\
\text { spp }\end{array}$ & H.indicus & $\begin{array}{l}\text { C. } \\
\text { limitaneum }\end{array}$ \\
\hline Control & $2.25 \mathrm{a}$ & $2.00 \mathrm{a}$ & $3.25 \mathrm{a}$ & $0.75 a$ & $0.5 \mathrm{a}$ & $1.00 a$ & $0.75 a$ & $0.50 a$ & $0.50 a$ \\
\hline CPP 100 & $1.25 \mathrm{ab}$ & $1.5 \mathrm{Oab}$ & $2.00 a b$ & $0.50 a b$ & $0.00 a$ & $0.50 a b$ & $0.75^{a}$ & $0.50 a$ & o.ooa \\
\hline $\mathrm{CPP}+\mathrm{NS}(9.1)$ & $1.25 \mathrm{ab}$ & $1.25 \mathrm{abc}$ & $1.75 \mathrm{ab}$ & $0.25^{a b}$ & $0.50 a$ & $0.75 \mathrm{ab}$ & $0.50 a$ & $0.00 a$ & $0.50 a$ \\
\hline $\mathrm{CPP}+\mathrm{NS}(8.2)$ & $1.5 \mathrm{Oab}$ & 1.5 oab & $2.00 a b$ & $0.25 \mathrm{ab}$ & $0.25 \mathrm{a}$ & $0.75 a b$ & $0.75^{a}$ & $0.25 a$ & o.ooa \\
\hline $\mathrm{CPP}+\mathrm{NL}(9.1)$ & $1.25 \mathrm{ab}$ & 1.ooabcd & $1.75 \mathrm{ab}$ & $0.25 \mathrm{ab}$ & $0.25 \mathrm{a}$ & $0.50 a b$ & $0.50 a$ & o.ooa & $0.00 a$ \\
\hline $\mathrm{CPP}+\mathrm{NS}(7.3)$ & $1.25 \mathrm{ab}$ & $0.75 \mathrm{bcd}$ & $1.5 \mathrm{ob}$ & o.oob & $0.50 a$ & $1.00 a$ & $0.75 a$ & o.ooa & o.ooa \\
\hline $\begin{array}{l}\mathrm{CPP} \\
(8.2)\end{array}+\mathrm{NL}$ & $1.25 \mathrm{ab}$ & $0.75 \mathrm{bcd}$ & $1.25 \mathrm{~b}$ & o.oob & $0.25 a$ & $0.25 \mathrm{ab}$ & $0.75^{a}$ & $0.00 a$ & $0.75 a$ \\
\hline $\mathrm{CPP}+\mathrm{NL}(7.3)$ & $1.00 a b$ & $0.25 \mathrm{~cd}$ & $1.5 \mathrm{ob}$ & o.oob & $0.25^{a}$ & $0.50 a b$ & $0.50 a$ & $1.50 a$ & $0.00 a$ \\
\hline Sterilized soil & $0.00 c$ & o.ood & $0.00 c$ & o.oob & $0.00 a$ & o.oob & o.oob & o.ooa & o.ooa \\
\hline
\end{tabular}

*Means followed by the same letter in the same column do not differ significantly according to Duncan Multiple Range Test (5\%).

Table 7: Effects of neem-fortified cassava peel soil amendments on the growth and yield of sugarcane in a screen house trial in 2013

\begin{tabular}{llllll}
\hline Treatment & Shoot wt(g)/plant & Root wt(g)/plant & Tiller no. & Height (cm) & Girth (mm) \\
\hline CPP+NL (70:30) & $862.50 \mathrm{a}$ & $247.50 \mathrm{a}$ & $1.75 \mathrm{a}$ & $172.67 \mathrm{a}$ & $18.83 \mathrm{a}$ \\
CPP+NL (80:20) & $742.50 \mathrm{c}$ & $226.75 \mathrm{~b}$ & $1.75 \mathrm{a}$ & $152.67 \mathrm{~b}$ & $18.65 \mathrm{a}$ \\
CPP+NS (70:30) & $726.25 \mathrm{~cd}$ & $217.75 \mathrm{c}$ & $1.75 \mathrm{a}$ & $116.17 \mathrm{c}$ & $16.98 \mathrm{~b}$ \\
CPP+NS (80:20) & $730.00 \mathrm{~cd}$ & $215.00 \mathrm{~cd}$ & $1.75 \mathrm{a}$ & $114.00 \mathrm{~cd}$ & $16.68 \mathrm{bc}$ \\
CPP+NL (90:10) & $725.00 \mathrm{~cd}$ & $208.75 \mathrm{de}$ & $1.75 \mathrm{a}$ & $111.67 \mathrm{~d}$ & $16.43 \mathrm{~cd}$ \\
CPP+NS (90:10) & $720.75 \mathrm{~cd}$ & $204.50 \mathrm{e}$ & $1.50 \mathrm{a}$ & $108.17 \mathrm{e}$ & $16.18 \mathrm{cde}$ \\
CPP 100 & $713.00 \mathrm{~d}$ & $205.00 \mathrm{e}$ & $1.50 \mathrm{a}$ & $102.83 \mathrm{f}$ & $16.13 \mathrm{de}$ \\
Sterilized soil & $628.75 \mathrm{e}$ & $195.25 \mathrm{f}$ & $1.50 \mathrm{a}$ & $93.83 \mathrm{~g}$ & $15.68 \mathrm{e}$ \\
Control & $528.75 \mathrm{f}$ & $173.25 \mathrm{~g}$ & $1.50 \mathrm{a}$ & $83.5 \mathrm{oh}$ & $14.85 \mathrm{f}$ \\
\hline
\end{tabular}

*Means followed by the same letter in the column do not differ significantly according to Duncan Multiple Range Test ( 5 \%)., $\mathrm{CPP}=$ Cassava Peel Powder, NL $=$ Neem Leaf Powder, NS $=$ Neem Seed Powder.

Table 8: Effects of neem-fortified cassava peel soil amendments on the growth and yield of sugarcane in a screen house trial in 2014

\begin{tabular}{llllll}
\hline Treatment & Shoot wt/plant (g) & Root wt/plant(g) & Tiller number & Height (cm) & Girth (mm) \\
\hline CPP+NL (70:30) & $884.00 \mathrm{a}$ & $250.83 \mathrm{a}$ & $2.67 \mathrm{a}$ & $179.5 \mathrm{a}$ & $17.28 \mathrm{a}$ \\
CPP+NL (80:20) & $761.17 \mathrm{abc}$ & $237.83 \mathrm{ab}$ & $2.67 \mathrm{a}$ & $152.67 \mathrm{~b}$ & $16.69 \mathrm{~b}$ \\
CPP+NS (70:30) & $746.17 \mathrm{bc}$ & $228.5 \mathrm{Ob}$ & $2.67 \mathrm{a}$ & $120.67 \mathrm{~b}$ & $16.43 \mathrm{c}$ \\
CPP+NS (80:20) & $737.67 \mathrm{~cd}$ & $223.67 \mathrm{bc}$ & $2.67 \mathrm{a}$ & $114.67 \mathrm{~cd}$ & $16.1 \mathrm{~d}$ \\
CPP+NL (90:10) & $733.33 \mathrm{~cd}$ & $212.17 \mathrm{~cd}$ & $2.67 \mathrm{a}$ & $116.00 \mathrm{c}$ & $16.43 \mathrm{c}$ \\
CPP+NS (90:10) & $714.00 \mathrm{~cd}$ & $204.33 \mathrm{de}$ & $2.67 \mathrm{a}$ & $114.00 \mathrm{~d}$ & $15.22 \mathrm{e}$ \\
CPP 100 & $683.67 \mathrm{de}$ & $194.33 \mathrm{ef}$ & $2.67 \mathrm{a}$ & $105.83 \mathrm{e}$ & $14.28 \mathrm{f}$ \\
Sterilized soil & $640.33 \mathrm{ef}$ & $184.5 \mathrm{Of}$ & $2.67 \mathrm{a}$ & $102.00 \mathrm{f}$ & $14.18 \mathrm{f}$ \\
Control & $610.33 \mathrm{f}$ & $166.00 \mathrm{a}$ & $2.50 \mathrm{a}$ & $91.67 \mathrm{~g}$ & $13.43 \mathrm{~g}$ \\
\hline
\end{tabular}

*Means followed by the same letter in the column do not differ significantly according to Duncan Multiple Range Test (5 \%).

\section{DISCUSSION}

Soil amendment with plant materials such as cassava peels and neem leaves are ancient practices that have been adopted by many local farmers to improve soil fertility without being fully aware of their potential nematicidal properties. The results of this investigation revealed that cassava peel powder singly and cassava peel powder in combination with either neem leaf or seed powder were not phytotoxic to sugarcane plants, rather they increased the nutrient status of the soil and subsequently led to an increase in the growth and yield of sugarcane in the amended soil. This is in agreement with the findings of [24] who reported that cassava peel amendment when applied singly and in combination with locust bean husk controlled population of Meloidygyne incognita on sugarcane and subsequently led to increased cane yield. It had also been indicated by [19] that a significant increase in the plant height and number of leaves per plant of cowpea cv. Ife brown on plots treated with both decomposed and un-decomposed cassava peels. The suppressive activity of the plant extracts could be linked to the possible release of nematotoxic and other antagonistic 
substances. The breakdown and mineralization of the plant extracts also result in the release of nutrients to the soil, thus improving the soil structure, fertility and increased microbial activities that promote restoration of soil integrity and eventually culminate in improved crop yield.

It was observed from the screenhouse trials that free living nematodes occurred in higher numbers in the soil treated with neem-fortified cassava peel powder while the reverse was recorded in the unamended soils where greater number of the plant-parasitic nematodes was recorded. This could be due to increased microbial activity that is often associated with decomposition of organic matter in the soil $[7,20,28]$.

The result of the present investigation shows that soil amendment with neem-fortified cassava peels resulted in a significant decrease in the soil and root density of plantparasitic nematodes that was recorded on sugarcane plants in the screen house trials and also a significant increase in the weight of top growth of the sugarcane plants. It was reported by [12] that root damage by nematodes resulted in a reduction of the number, length, diameter of the stalk and sucrose content of sugarcane. Similar report had also been given by [10] who stated that mulching or application of well decomposed cattle or poultry manure, compost or neem oil cake can reduce nematode build up.

Cassava contain a large amount of prussic acid ( $\mathrm{HCN})$, with greater concentration in the phelloderm [13] the function of the glucosides in cassava was that of liberating hydrogen cyanide, which is effective in repelling insect pest that attacked the plant. The suppression of plant-parasitic nematodes by cassava peels powder as indicated by the result of this study could be as a result of toxic metabolites [17]. Neem contains bioactive principles that belong to a class of compounds known as limonoids of which Azadirachtin is important [3, 20, 25]. Neem contains insect growth inhibitors $[26,8]$. It also indicated by [23] that $A$. indica as the most effective nematicidal plant for nematode control in tomato

There is a great likelihood that the addition of neem to the cassava peels will further improve and reinforce the nematotoxic potential of the organic material thereby resulting in greater nematode suppression [25]. This is evident in the result of this present investigation where higher nematode suppression was recorded on sugarcane plants with neem-fortified cassava peel as compared to those that were amendment with cassava peel alone. Evidence of decrease in nematode population by neem leaves fertilization has been reported [30, 4, 21, 32]

\section{CONCLUSION}

The key to sustainable agricultural production depends on the maintenance of soil integrity. Excessive use of synthetic pesticides, herbicides and even fertilizers has greatly compromised the soil ecosystem with heightened concerns on the long term detrimental effects on human health and the environment. Therefore, soil amendment with neem-fortified cassava peel is recommended as a potential alternative to synthetic nematicides in management of nematode pests of agricultural crops.

\section{REFERENCES}

1. Abdul-latif, D. Busari, 2004. Sugarcane and Sugar Industry in Nigeria. The bitter-sweet lessons. Pp 6-66

2. Aktah, A, and A. Malik, 200o. Roles of organic soil amendment and soil organisms in the biological control of plant-parasitc nematodes: A reviw. Bioresource Tech. pp74-34.

3. Akhtar, M., 2000. Nematicidal potential of the neem tree Azadirachta indica (A. Juss). Integrated Pest. Manag. Rev. 5:57-66.

4. Akhtar, M. and M. Alam, 1990. International nematology network news letter 7:10.

5. Amadioha, A. C., 2003. Evaluation of some plant leaf extracts against Colletotrichum lindemuthianum in cowpea. Actaphyto pathologica et Entomologica Hungarica, 38 (3-4): 259-265.

6. Badra, T., Saleh, M. A. and B. A. Oteifa, 1979. Nematicidal activity and composition of some organic fertilizers and amendments. Revue de Nematologie, 2:29-36.

7. Chirchir, A. K., J. W. Kimenju, F. M. Olubayo and G. K. Mutua, 2008. Abundance and Distribution of Plant Parasitic Nematodes Associated with Sugarcane in Western Kenya. Asian Journal of Plant Pathology, 2:48-53.

8. D'Addabbo T, Laquale S, Lovelli S, Candido V, Avato $P$. Biocide plants as a sustainable tool for the control of pests and pathogens in vegetable cropping systems. Italian Journal of Agronomy. 2014;9(4):137-45.

9. Dinardo-Miranda, L. L and J. V. Fracasso, 2009. Spatial distribution of plant-parasitic nematodes in sugarcane fields. Sci. Agric. (Piracicaba, Braz.), v.66, n.2, p.188-194

10. Dohroo, N. P, Sharma, O., Sharma, M. and R. S. Sarlach, 1994. Effect of organic amendment of soil on rhizome rot, nematodes and rhizosphere mycoflora of ginger (Zingiber officinale Roscoe). Annas of Biology, Ludhiana 10, 208-210.

11. Egunjobi A. O. and S. O. Afolami, 1976. Effect of neem (Azadirachta indica L.) leaf extracts on population of Pratylenchus brachyurus and on the growth and yield of maize Nematologica 22:125-132.

12. Egunjobi, A. O. and G. O. Ekundare, 1981. Cassava peelings as soil amendment and its effects on maize yield in soil infested with Pratylenchus brachyurus, Nigeria Journal of Crop Plant Protection 5:80-87

13. Famurewa, J. A. V., and P. O. Emuekele, 2014. Cyanide reduction pattern of cassava (Mannihot esculenta) as affected by variety and air velocity using fluidized bed dryer. Afr. J. Food Sci. Technol. 5:75-80

14. FAO 2004 FAO Statistic Division. www. faostat. org

15. Hartmann, Martin, Beat Frey, Jochen Mayer, Paul Mäder and Franco Widmer, 2014. Distinct soil microbial diversity under long-term organic and conventional farming The ISME Journal advance doi: 10.1038/ismej.2014.210

16. Mai W. F. and H. H. Lyon, 1975. Pictoral key to Genera of Plant-parasitic nematodes, 4th Edition. Cornerstone Publishing Associates. A Division of Cornell University Press, pp.219.

17. Michel LUC, Sikora, R. A and J. Bridge, 2005. Plant Parasitic Nematodes in Subtropical and Tropical Agriculture. $2^{\text {nd }}$ Edition. 493-520.

18. National Sugar Development Council, 2003. Information Brochure towards Self Sufficiency in Sugar, Abuja. pp l-26

19. Olabiyi, T. I., Akanbi W. B and I. O Adepoju 2007. Control of certain nematode pest with different organic manure on cowpea. American-Eurasian Journal of Agriculture and Environment Science, 2: 523-527

20. Oka, Y., Tkachi N, Shuker S, and U. Yerumiyahu, 2007. Enhanced nematicidal activity of organic and 
inorganic ammonia-releasing amendments by Azadirachta indica extracts. J. Nematol. 39:9.

21. Oka, Y. and U. Yermiyahu, 2002. Suppressive effects of composts against the root-knot nematode Meloidogyne javanica on tomato. Nematology $4: 891^{-}$ 898.

22. Okonkwo., E. M., Ladan, Z., Agbaji, A. S., Booth, S. A., Falodun, S. and A. AYusuf, 2008. Pilot-Scale Production of neem-based bio-agro inputs for organic agriculture in Nigeria pp 43-45. In proceedings of 1st West African Summit and 4th National Conference on Organic Agriculture 17-21 November, 2008 University of Agriculture, Abeokuta, Nigeria Aiyelaagbe (eds I. O. O., Adetunji, M. T. and Osei, S. A) 236 p

23. Randhawa N., Sakhuja P. K., and I. Singh, 2001. Management of rootknot nematode Meloidogyne incognita in tomato with organic amendments. Plant Dis. Res. 16:274-276.

24. Salawu EO. Effects of cassava root peeling and locust bean husk amendments in controlling Meloidogyne incognita of sugarcane (Saccharum officinarium L.). Plant Protection in Tropics. 1988; 5: 11-5.

25. Salawu, E. O., 1992. Effect of neem leaf extract and ethoprop singly and in combination on Meloidogyne incognita and growth of sugarcane. Pakistan Journal of Nematology 10, 51-56.

26. Schmutterer, H., 1990. Properties and Potential of natural Pesticides from the neem tree, Azadirachta inidica. Annu. Rev. Entomol., 35:271-97, ISSN: 00664170 .
27. Shivashankar, S., T. K. Roy and P. N. Krishna Moorthy, 2012. Headspace Solid Phase Micro Extraction and GC/MS Analysis of the Volatile Components in Seed and Cake of Azadirachta indica A. juss. Chem. Bull. "POLITEHNICA" Univ. (Timisoara) Volume 57, 1, 2012

28. Sikora, R. A., Schafer, K. And A. A Dababat, 2007. Modes of action associated with microbially induced in plants suppression of plant parasitic nematodes. Australasian plant pathology, 36:124-134

29. Spaull V. W. and P. Cadet, 1991. Nematode Parasites of Sugar cane. In: Plant-Parastic Nematodes in subtropical and tropical Agriculture. (eds. M. LuC, R. A Sikora and J. Bridge) P.461-491. Wallingford, U. K CAB International.

30. Stirling, G. R., 1991. Biological control of plantparasitic nematodes. Cab international, Wallingford, UK. 275p.

31. Tomerlin, A. H., Arhur, H. and G. C. Smari, 1969. The influence of organic soil amendments on nematodes and other soil organisms. Journal of Nematology 1:2930 (abtr.)

32. Walker, G. E., 2004. Effects of Meloidogyne javanica and organic amendments, inorganic fertilizers and nematodes on carrot growth and nematode abundance. Nematologia Mediterranea 32 : 181-188.

33. Whitehead, A. F., and J. R. Hemming 1965. A comparison of some quantitative methodes of extracting semi veriform nematodes from soil. Annals of Applied Biology . 55:25-28. 